Special issue of the 3rd International Conference on Computational and Experimental Science and Engineering (ICCESEN 2016)

\title{
Influence of Cavity Design on Stress Distribution in Second Premolar Tooth Using Finite Element Analysis
}

\author{
Z. PARlaR ${ }^{a, *}$,E.U. GÖKÇEK ${ }^{a}$, K. Yildirim ${ }^{a}$ AND A. KAHYAOGLU ${ }^{c}$ \\ ${ }^{a}$ İstanbul Technical University, Mechanical Engineering Fakulty, Ístanbul, Turkey \\ ${ }^{b}$ Dentist, İstanbul, Turkey \\ ${ }^{c}$ Arzu Kahya, Dentist, PhD, Private Practice, İstanbul, Turkey
}

\begin{abstract}
The objective of this study is, using finite element analysis, to design cavity geometry, to minimize risk of the fraction and cracking in second premolar tooth. To avoid possible tooth fracture, proper cavity geometry can be designed, to reduce the effect of masticatory forces. Premolar tooth was chosen due to its least strength in comparison to other type of teeth. In literature, there was insufficient study on using finite elements methods to investigate restorated premolar teeth. A healthy premolar tooth was scanned to obtain 3D model of it, to perform finite elements analysis. The validation was performed based on existing experimental data. According to stress distribution, three different cavity geometries were designed using composite resin and ceramic as a restoration material. It is observed that the amount of stress acting on tooth is diminishable with regard to analysis results. Hence, proper cavity geometry and material were found to minimize the risk of tooth fracture.
\end{abstract}

DOI: 10.12693/APhysPolA.132.949

PACS/topics: 45.10.-b, 46.25.--y

\section{Introduction}

The loss of material and strength of teeth due to caries are among principal issues faced by dentists and odontologists. Relevant research was focused on reducing respective damage through implementation of different restorative materials and cavity geometry $[1,2]$. Numerical analyses have been performed to obtain stress distribution using 3D finite element model [3-6]. However, in spite of the effort and relatively successful implementation of restorative materials that convey lower stresses on the tooth, there had been no significant breakthrough in this regard. Studies on different cavity geometries also proved nonproductive due to the unpredictability of caries forms.

In present study, a finite element model of a 3D scanned tooth subject to simulated masticatory forces is used to carry out stress distribution analysis in various conditions, for premolar tooth. Finite element models that are easily modifiable and able to provide quick results become more and more prominent with the advances in finite element software capabilities.

The difficulties in procuring healthy teeth and teeth restored with different cavity geometries or restoration materials, as well as high costs of such endeavor, cause a significant restriction in experiment cases, providing limited results. Therefore, creating cavity geometries and restoration material cases for both composite and ceramic materials in a simulation environment in order to cover more cases in an experimental matrix proves a viable solution.

*corresponding author; e-mail: parlarze@itu.edu.tr

\section{Materials and methods}

The radiology is a common method for diagnosis and treatment [7]. Using radiation increases cancer risk of both, patients and medical staff $[8,9]$. For that reason, dental restorative material properties such as radioactivity, hardness and elastic modulus are important for patient's health and long life restoration [10-12].

Composite and ceramic materials that are more popular among restorative materials, with their natural looks and good mechanical properties, were chosen as material variants for this study. Basic mechanical properties for common restorative materials are provided in Table I.

TABLE I

Basic mechanical properties of restorative materials [13].

\begin{tabular}{c|c|c}
\hline \hline Structure/material & $\begin{array}{c}\text { Elastic modulus } \\
{[\mathrm{MPa}]}\end{array}$ & Poisson ratio \\
\hline Enamel & $46.8 \times 10^{3}$ & 0.30 \\
Dentin & $18.0 \times 10^{3}$ & 0.31 \\
Composite resin & $16.6 \times 10^{3}$ & 0.24 \\
Ceramic inlay & $65.0 \times 10^{3}$ & 0.23
\end{tabular}

First step of the study is the reconstruction of a healthy unrestored premolar. A human maxillary second premolar, extracted for orthodontic reasons was used for this purpose. The selected tooth was intact, without caries, fracture or morphological abnormalities. The 3D model of the tooth was generated through 3D laser scanning. Reconstruction artifacts on the scanned structure were fixed with a commercial CAD software and the model was divided in three layers representing pulpa, enamel and dentin, as shown in Fig. 1. The layers exhibiting the properties of their respective structures were assumed to be perfectly bonded to each other and modeled as such. 
The boundary conditions were specified to be consistent with physical conditions. The root of the modals was set as fixed along $x, y$ and $z$ directions, for all of the studied cases. Thus, deflection near the root was minimized. The model was exported into the finite analysis software and translated into a mesh structure by an integrated volumetric 3D mapping algorithm, as shown in Fig. 2. The finite element mesh consists of 95812 nodes and 54416 quadratic elements.

Tooth layer materials were assumed to be isometric, neglecting the anisotropic properties of dentin and enamel materials. Hence, the entire tooth structure and all materials used in the models were considered isotropic, elastic, linear and homogeneous [14]. Equivalent stress values were calculated using Von-Mises equation, which produces good approximations to experimental results [5]. Finite element analyses were performed using ANSYS software.

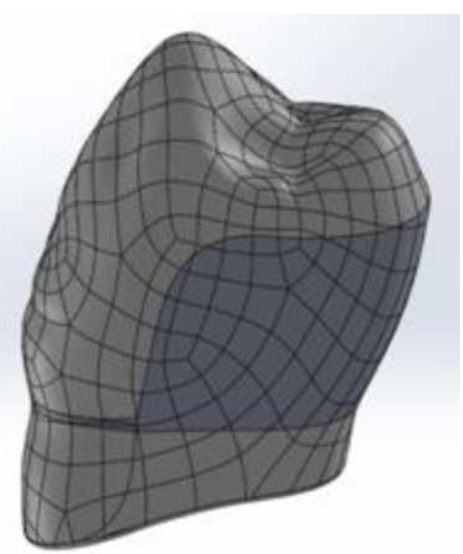

Fig. 1. Healthy tooth model with pulpa, dentin and enamel layers.

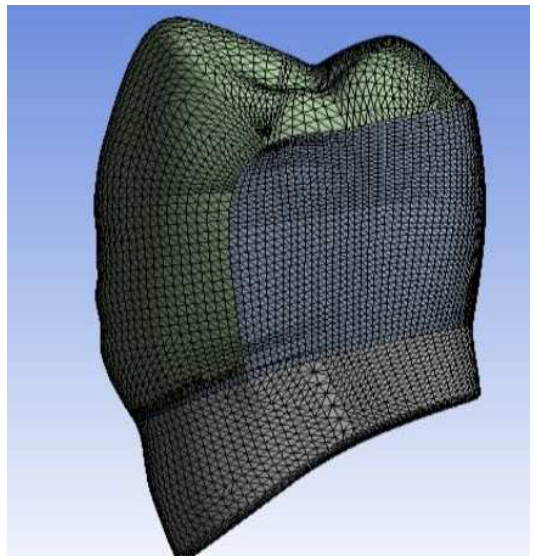

Fig. 2. Generated mesh.

\section{Validation process}

This study does not involve an experimental step, due to ethical issues in procuring real tooth samples. Instead, the analysis results of this study were compared to previous experimental outcomes on healthy premolars presented by Soares et al. [13]. For this purpose the premolar model was exposed to oblique forces acting perpendicularly to the inner cusp inclines, which lay at approximately $45^{\circ}$ to the vertical loading axis. The force was equal to $90 \mathrm{~N}$ and assumed as broken down into two force vectors of $45 \mathrm{~N}$, applied to the tooth surface. The definition of forces and stress distribution of healthy tooth are shown in Fig. 3.
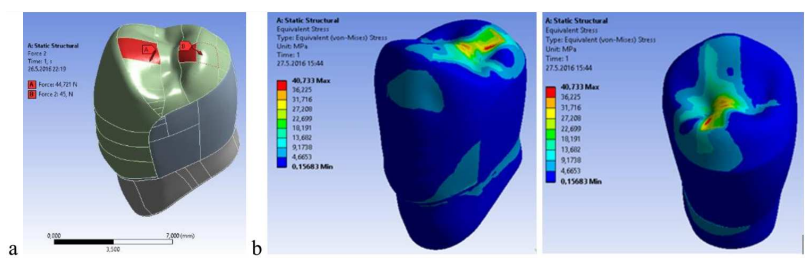

Fig. 3. (a) Definition of forces, (b) stress distribution.

Maximum stress value is approximately $40 \mathrm{MPa}$. There are some discrepancies between numerical results and experimental data due to differences between the force acting points and assumptions for the simulation model. Another factor is anatomical tooth variability, since a large number of anatomic details may alter the generated stress.

Burke et al. classified four fracture modes as follows [15]:

- Mode I fractures, involving a small portion of the coronal tooth structure.

- Mode II fractures, involving a small portion of the coronal tooth structure and cohesive failure of the restoration.

- Mode III fractures, involving the tooth structure, cohesive and/or adhesive failure of the restoration, with root involvement that can be in association with periodontal surgery.

- Mode IV severe root and crown fracture, which require extraction of the tooth.

The mentioned fracture modes point out stress concentrations in certain regions of tooth, resulting in fractures. Figure $3 \mathrm{~b}$ reveals stress concentrations in the same regions. This consistency is considered to support the validity of the model.

\section{Results}

All performed analyses were linear static. A standard Class II MOD cavity was modeled as a reference in our search for a better performing cavity model. This reference model is presented in Fig. 4a. Proximal regions of the cavity were kept in the confines of the enamel layer. The initial analyses were carried out on this common geometry and following models were designed according to 
the stress distribution obtained in the original one. Two different restoration materials were considered to gain insight on the effects of materials on stress distribution. The models were analyzed for both, composite resin and ceramic materials. Changes in material characteristics due to morphological variations of cavity structures were also considered [16].

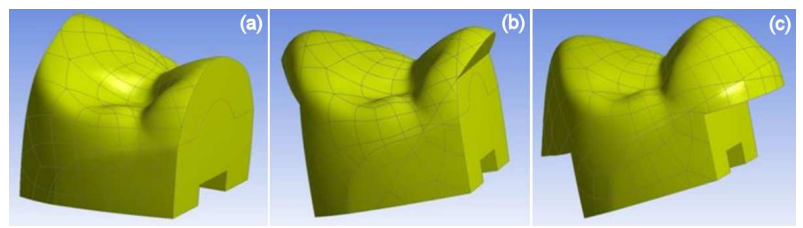

Fig. 4. Cavity models: (a) Class II MOD, (b) Design I cavity, (c) Design II cavity.

One of the main objectives in designing alternative cavity geometries is minimizing clinically observed postrestoration tooth fractures by obtaining a more homogeneous stress distribution along with lower maximum stress. For this purpose, Design I cavity was developed with additional tooth material removal beveled at $45^{\circ}$ at the borders of the enamel layer, as shown in Fig. 4b. Both vertical and horizontal force components are present as a result of a $45^{\circ}$ angle between the applied force and the vertical loading axis. It should be noted that the horizontal components act towards the separation of buccal and palatal surfaces. Design II Cavity was created with the aim of counteracting this effect with $90^{\circ}$ horizontal tooth relief, extending to tubercles, as shown in Fig. 4c.

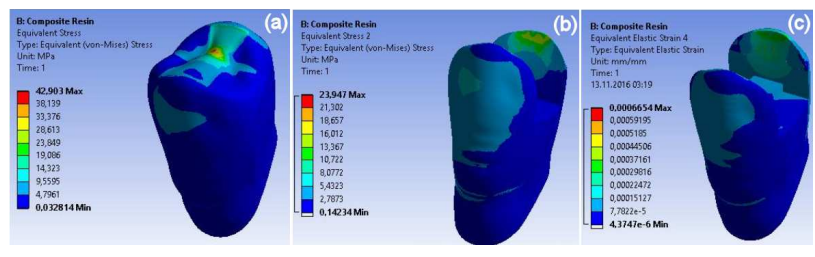

Fig. 5. Stress analysis for Class II MOD cavity restored with composite resin: (a) complete model, (b) tooth structure, (c) strain distribution in tooth structure.

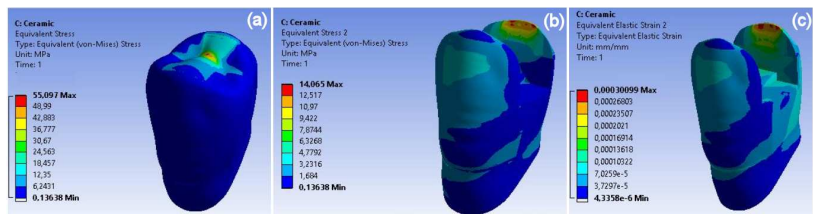

Fig. 6. Stress analysis for Class II MOD cavity restored with ceramic: (a) complete model, (b) tooth structure, (c) strain distribution in tooth structure.

Figures 5 and 6 reveal the stress distribution results for composite resin and ceramic inlay, respectively. It can be observed that stress concentrations generally occur close to the root and the interface between the tooth and the restorative material. In case of composite resin, a maximum stress value of $43 \mathrm{MPa}$ occurs in the restoration, while the maximum stress value conveyed to the tooth remains at $24 \mathrm{MPa}$. The strain value around the cusp is approximately $0.66 \mu \mathrm{m}$. Respective values for ceramic inlays are $55 \mathrm{MPa}, 14 \mathrm{MPa}$ and $0.3 \mu \mathrm{m}$.

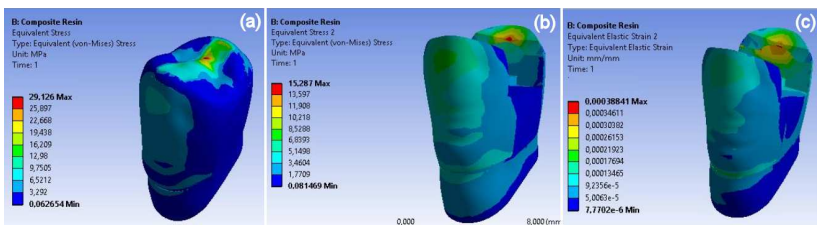

Fig. 7. Stress analysis for Design I cavity restored with composite resin: (a) complete model, (b) tooth structure, (c) strain distribution in tooth structure.

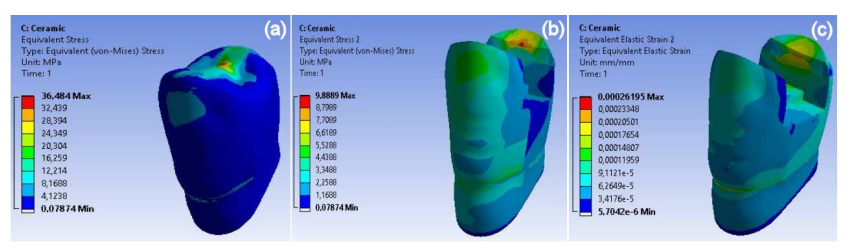

Fig. 8. Stress analysis for Design I cavity restored with ceramic: (a) complete model, (b) tooth structure, (c) strain distribution in tooth structure.

Figures 7 and 8 present the stress distributions for composite resin and ceramic inlay, respectively. Design I presents lower stress values compared to Class II MOD cavity. Moreover, a more homogeneous distribution is observed on the buccal and palatal surfaces, as expected, while no significant change in strain is observable. Respective on-tooth maximum stress values for composite resin and ceramic inlay are $15 \mathrm{MPa}$ and $10 \mathrm{MPa}$.

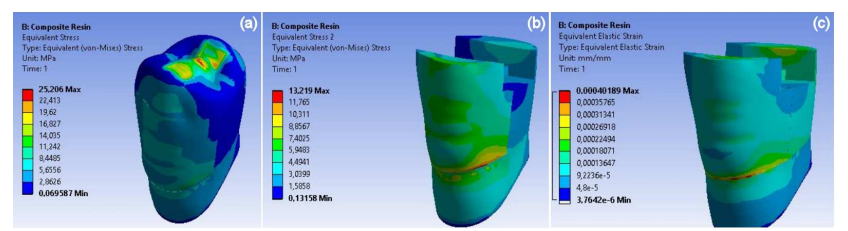

Fig. 9. Stress analysis for Design II cavity restored with composite resin: (a) complete model, (b) tooth structure, (c) strain distribution in tooth structure.

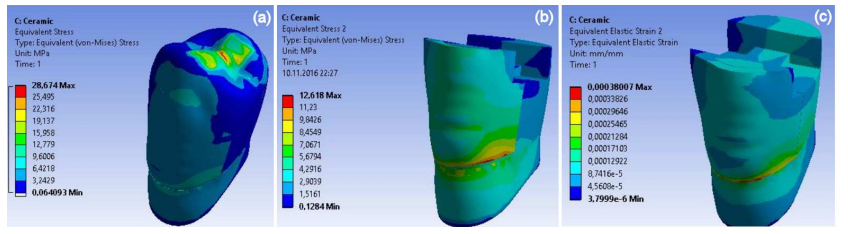

Fig. 10. Stress analysis for Design II cavity restored with ceramic: (a) complete model, (b) tooth structure, (c) strain distribution in tooth structure.

The stress distribution analysis results for Design II Cavity are presented in Figs. 9 and 10 for composite resin and ceramic inlays, respectively. Resulting stress distributions are observed to be fairly homogeneous at tooth surface for both materials, although stress concentrations are present at the intersection of the root and the 
crown. Approximate stress at this point for the tooth restored with composite resin is $13 \mathrm{MPa}$, while the one for the tooth, restored with ceramic, stands at $12 \mathrm{MPa}$. The strain around the cusp is minimal, on account of the elimination of horizontal force components.

The equivalent maximum stress and strain values for each case are listed in Table II. According to the results presented in Table II, both restorative materials yield relatively high equivalent stress values in case of this reference model. On the other hand, the extra tooth relief, introduced in Design I cavity wall, causes a significant decrease in equivalent stress. Even though Design II with horizontal tubercle relief results in higher stress values, compared to Design I, it still stands out as an option that eliminates the concerns for leakage at tooth and restoration interface, introduced by the previous model.

TABLE II

Analysis results for different cavity designs and different restorative materials.

\begin{tabular}{c|c|c|c|c|c|c|c|c}
\hline \hline \multirow{2}{*}{} & \multicolumn{4}{|c|}{ Equivalent stress [MPa] } & \multicolumn{2}{c}{$\begin{array}{c}\text { Equivalent max. strain 10 } \\
{[\mathrm{mm} / \mathrm{mm}]}\end{array}$} \\
\cline { 2 - 8 } & \multicolumn{2}{|c|}{ Composite } & \multicolumn{2}{c|}{ Ceramic } & \multicolumn{3}{c}{ Composite } & \multicolumn{2}{c}{ Ceramic } \\
\cline { 2 - 8 } & Restoration & $\begin{array}{c}\text { Tooth } \\
\text { structure }\end{array}$ & Restoration & $\begin{array}{c}\text { Tooth } \\
\text { structure }\end{array}$ & cusp & root & cusp & root \\
\hline Class II MOD & 43 & 24 & 55 & 14 & 3.72 & 2.25 & 3.01 & 2.85 \\
Design I & 29 & 15 & 36.5 & 10 & 3.46 & 1.77 & 3.84 & 2.05 \\
Design II & 25 & 13 & 29 & 13 & 3.55 & 4.02 & 2.57 & 3.80
\end{tabular}

A closer observation of the cross-section of restored teeth allows us to make a decision between Design I cavity and Design II cavity, both of which yield maximum stress values close to each other. A thorough evaluation of the cross-sections reveals that Design I provides a more homogeneous stress distribution, rendering it the better design option, considering the small difference between their maximum stress values. Analysis results show that maximum strain in cusp is the lowest in Design II for ceramic inlays and is highest in the class II MOD reference design. On the other hand, the lowest equivalent strain values are observed for Design I composite resin, at the root.

\section{Discussion}

A 3D solid tooth model was created using 3D laser scanning methods. Realistic modeling allows the researchers to calculate general stress distribution across the model and to predict possible fracture modes $[2,3,6]$. Furthermore, computational methods can be carried out with much lower costs, compared to experimental studies and clinical trials. However, we still face persistent challenges, as a complete simulation of the oral environment is still far from our reach. After all, numerical analysis has an important role to produce novel ideas for clinical operations. More recent FEM studies have evaluated several parameters of occlusal forces, MOD cavity preparation geometry and dental materials, used to restore maxillary premolar teeth [17-19].

The primary objective of modern restoration applications in dentistry is the preservation of healthy tooth. Restorative tooth surgery focuses on protecting the remaining tooth structure from unfavorable mechanical responses, even if there is a necessity of removing additional dental tissue [20]. Fracture of the cusp of a tooth is a common clinical issue in such applications. Soares et al. have experimentally confirmed that palatal cusp-related fractures are more severe than buccal cusp-related fractures [21]. Stresses concentrate intensively on the cusps due to oblique occlusal forces and such occurrences increase the risk of tooth fracture [17].

A similar observation was made in this study with high stress concentrations around the palatal cusps in Class II MOD and Design I restorations. The beveled Design I cavity provides $40 \%$ and $30 \%$ lower maximum stress for composite resin and ceramic inlay respectively, in comparison with Class II MOD. Lowest maximum stress is observed in ceramic inlays. Design II cavity prevents stress concentration on the cusps, owing to tooth forces acting normally and registers stress concentrations at the interface between the root and the crown. Such an application would be favorable for preventing cusp fractures, resulting from extensive crown damage. Endodontic treatment can weaken the tooth structure because of a deep and extended cavity, reducing the amount of dentin. Therefore, Design I cavity composite resin can be suggested for endodontic treatment in terms of root fracture prevention, with the lowest root strain value. Examination of the cusp and root strain shows that the lowest strain value is obtained for Design I composite resin at root and in Design II ceramic inlay at cups. Thus, when the tooth has excessive crown damage, Design II ceramic restoration should be considered to prevent cusp fracture.

\section{Conclusions}

In this study, the influence of the cavity design and restoration material on stress distribution for premolar tooth was investigated by way of finite element method. 
The lowest maximum equivalent stress value was observed in Design I restoration with ceramic inlay. Moreover, Design I stands out with significantly lower root strain, making it the best choice for root fracture prevention. On the other hand, Design II proved superior on prevention of crown fracture with lower stress on the cusps. In case of maximum stress value being the main concern, the lower cost of composite restoration could be the deciding factor, due to the good performance of both in this regard.

\section{References}

[1] L. Sandu, F. Topalâ, S.C. Porojan, Int. J. Med. Health Biomed. Bioeng. Pharmaceutical Eng. 11, 342 (2012).

[2] K. Zelic, A. Vukicevic, G. Jovicic, S. Aleksandrovic, N. Filipovic, M. Djuric, Int. Endodontic J. 48, 850 (2015).

[3] D. Parle, A. Ambulgekar, K. Gaikwad, Simul. Driven Innovat., 1 (2012).

[4] Z. Huang, Z. Chen, J. Huazhong Univ. Sci. Technol. 33, 775 (2013).

[5] G. Milewski, Acta Bioeng. Biomech. 7, 47 (2005).

[6] D.Y. Lim, H.C. Kim, B. Hur, K.H. Kim, K. Son, J.K. Park, J. Kor. Acad. Cons. Dent. 34, 69 (2009).

[7] U. Kara, A. Mesbani, I. Akkurt, Acta Phys. Pol. A 128, B-372 (2015).

[8] U. Kara, D. Yildirim, I. Akkurt, Acta Phys. Pol. A 130, 407 (2016).
[9] U. Kara, I. Akkurt, Acta Phys. Pol. A 130, 404 (2016).

[10] I. Akkurt, R.B. Ermiş, P. Bas, K. Gunoglu, Acta Phys. Pol. A 128, B-34 (2015).

[11] Z. Arslanoglu, H. Altan, E. Kale, F. Bilgic, O. Sahin, Acta Phys. Pol. A 130, 388 (2016).

[12] H. Altan, F. Bilgic, Z. Arslanoglu, E. Kale, A.K. Kale, O. Sahin, Acta Phys. Pol. A 130, 394 (2016).

[13] P.V. Soares, P.C.F. Santos-Filho, H.A. Gomide, C.A. Araujo, L.R.M. Martins, C.J. Soares, J. Prosthetic Denstistry 99, 114 (2008).

[14] R.G. Craig (Ed.) Restorative Dental Materials, 9th ed., C.V. Mosby, Saint Louis 1993.

[15] F.J. Burke, J. Dent. 20, 131 (1992).

[16] B. Dejak, A. Mlotkowski, J. Prosthetic Denstistry 99, 131 (2008).

[17] M.L. Mei, Y.M. Chen, H. Li, C.H. Chu, BioMed. Eng. OnLine 15, 3 (2016).

[18] I. Kantardžić, D. Vasiljević, L. Blažić, O. Lužanin, Croat. Med. J. 53, 568 (2012).

[19] P. Ausiello, A. Apicella, C.L. Davidson, S. Rengo, J. Biomech. 34, 1269 (2001).

[20] C.L. Lin, Y.H. Chang, P.R. Liu, J. Dent. 36, 194 (2008).

[21] P.V. Soares, P.C. Santos-Filho, L.R. Martins, C.J. Soares, J. Prosthet. Dent. 99, 30 (2008). 